\title{
Interstrip Characteristics of n-on-p FZ Silicon Detectors
}

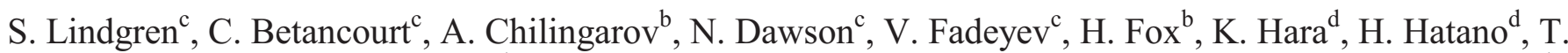

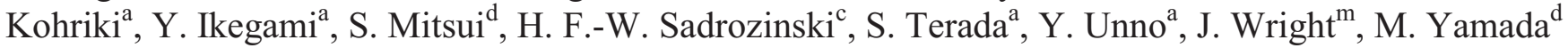 \\ ${ }^{a}$ KEK, High Energy Accelerator Organization, INPS, 1-1 Oho, Tsukuba, Ibaraki 305-0801, Japan \\ ${ }^{\mathrm{b}}$ Physics Department, Lancaster University, Lancaster LA1 4YB, United Kingdom \\ ' SCIPP, UC Santa Cruz, CA 95064, USA \\ ${ }^{\mathrm{d}}$ School of Pure and Applied Sciences, University of Tsukuba, 1-1-1 Tennodai, Tsukuba, Ibaraki 305-8571, Japan
}

\begin{abstract}
We report on the measurement of interstrip parameters of p-type silicon strip sensors which we are developing in a large collaboration to be used in a future tracker for the LHC upgrade. We measure on test structures with about 1 cm long strips the interstrip resistance, interstrip capacitance (at $1 \mathrm{MHz}$ ) and punch-through protection both pre-rad and after irradiation with $70 \mathrm{MeV}$ protons to a fluence of $1.5^{*} 10^{\wedge} 13$ $\mathrm{p} / \mathrm{cm}^{\wedge} 2$, corresponding to about $1 \mathrm{MRad}$, from prototyping runs with Hamamatsu Photonics and Micron Semiconductors. We report the values for a variety of isolation scenarios of $p$-stops, $p$ spray and a combination of both.
\end{abstract}

\section{INTRODUCTION}

$\mathrm{I}_{\mathrm{f}}^{\mathrm{N}}$ the need for radiation-hard tracking detectors in forthcoming elementary particle physics experiments, silicon is regarded to be the best choice as sensor material because of its unsurpassed material quality, mature technology and low cost for mass production [1]. Recently, a luminosity upgrade to $10^{35} \mathrm{~cm}^{-2} \mathrm{~s}^{-1}$ has been proposed to the Large Hadron Collider (LHC) ("SuperLHC") [2]. To exploit the physics potential of the upgraded LHC, an efficient tracking down to a few centimeters from the interaction point will be required, where fast hadron fluences above $10^{16} \mathrm{~cm}^{-2}$ will be reached after five years operation [3]. The CERN-RD50 project "Development of Radiation Hard Semiconductor Devices for Very High Luminosity Collider" has been formed to explore detector technologies that will allow to operate devices up to, or beyond, this limit [4], [5], and the two large all-purpose experiments ATLAS and CMS have started to plan for an upgrade of their detectors to exploit the expected higher luminosity.

One of the most pressing issue for $n-i n-p$ strip sensors are the interstrip characteristics before and after ionizing radiation, since the electron accumulation layer on the surface needs to be compensated for large fluences and dose levels. Both neutrons and protons displace silicon atoms via non-ionizing energy losses, which results in a bulk damage. Protons in addition ionize the atoms in their path that leads to permanent damage at the sensor surface, which makes them the main radiation species to study for surface damage.

The interstrip resistance and capacitance are important parameters used to characterize the effects of surface radiation damage of silicon strip detectors. The interstrip resistance is important for strip isolation, so that a sufficiently high interstrip resistance can prevent signal sharing between neighbors which could lead to degradation of the position resolution. The interstrip capacitance is the main contributor of noise in between strips. A properly functioning detector should thus try to minimize the interstrip capacitance in order to have a higher signal-to-noise ratio, while maximizing the interstrip resistance to minimize crosstalk between strips.

Ac-coupled sensors are susceptible to very large voltages between the metal readout traces (held to ground through the front-end electronics) and the strip implants in the case of large charge accumulation in the bulk, for instance in the case of beam losses [6]. Since the field inside of the sensor breaks down, these large voltages on the implants can reach the order of half the bias voltage, and thus can exceed the specification for the hold-off voltage of the coupling capacitor, which are typically tested to $100 \mathrm{~V}$. In order to prevent these large voltages, the punch-through (reach-through) effect is used [6], where implants in close proximity will effectively be shorted together if the voltage between them exceeds a geometry dependent voltage. This provides an effective over-voltage protection for single strips, which get shorted to the bias line in the case of voltages in excess of the punch-though protection ("PTP") voltage.

Previous studies with p-type sensors, e.g. within the context of RD50, were done using p-spray to isolate the n-strips. This study uses "mini-SSDs" ( $\sim 1 \mathrm{~cm}$ long) produced by Hamamatsu Photonic (HPK) within the ATLAS upgrade program. The isolation is done with $\mathrm{p}$-stops of varying geometry, $\mathrm{p}$-spray and both combined with $\mathrm{p}$-doses (concentration) varying from 0 up to $2 * 10^{13} \mathrm{p} / \mathrm{cm}^{2}$.

This paper is presented as follows. Section II describes the samples and irradiation. Section III describes interstrip capacitance and resistance measurements. Section IV describes punch-through protection. Section V presents results. Section VI presents conclusions as well as discussion.

\section{SAMPLES AND IRRADIATION}

The sample sensors were fabricated using $15 \mathrm{~cm}$ wafers with $<100>$ crystal orientation and $320 \mu \mathrm{m}$ thickness. The wafers we report in this paper are FZ grown (FZ p wafers in [7]) having fewer defects than normal FZ wafers. The R\&D group continues to evaluate other commercially available p-type wafers [7]. The strip pitch is $74.5 \mu \mathrm{m}$. The characteristics of irradiated sensors are studied using miniature samples of 10 $\mathrm{mm}$ square, where there are 104 strips of $8 \mathrm{~mm}$ length. 


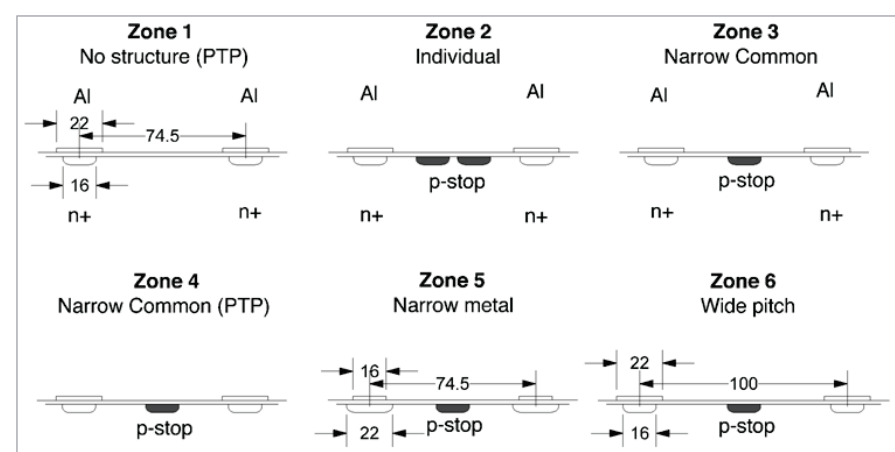

Fig. 1. Different $\mathrm{p}$-stop structures as indicated by the different zone numbers

It is thought that $n$-on-p detectors are more sensitive to surface effects than p-on-n detectors. One concern is the risk that the fixed oxide charges in the $\mathrm{Si}-\mathrm{SiO}_{2}$ interface would lead to a conductive layer of electrons at the surface [8]. Within the project ATLAS07 for the ATLAS upgrade different structures for mini-SSDs have been produced. The different structures use the concept of preventing those damages by surface treatments, positive doped implants (p-impurities) in form of p-stop or p-spray, or combinations of both.

The p-stops are implanted to the detectors with a mask while p-spray is sprayed on. Several doses and combinations of $p$ stop and p-spray have been applied to different sensors. Different structures to apply the p-stops have also been used, which is indicated by different zone number and seen in Fig. 1.

Detectors with zone 1 have no structure, i.e. they have only p-spray since the p-stop mask was left out. Detectors with zone 2 have individual p-stops, i.e. each strip is surrounded by pimplants in opposite to the other structures which only have a line of common p-implants between the strips. Zone 3 shares the p-stops between the strips and zone 4 has additional punchthrough protection structure, which will be discussed more later in the paper. Detectors with zone 5 have narrow metal, meaning the aluminum layer over the strips do not reach outside the strip itself and finally zone 6 is similar to zone 3 but with a wider strip pitch.

The proton irradiation was performed at Cyclotron Radio Isotope Center (CYRIC) of Tohoku University. Details of irradiation facility and methods are described in [7,9]. 70$\mathrm{MeV}$ protons were uniformly irradiated by scanning periodically the sample sensors. The irradiation took typically a few 10 minutes to a few hours depending on the fluence. The sensors were kept cooled at $-10^{\circ} \mathrm{C}$ during irradiation and the irradiated samples were immediately stored in refrigerator to suppress any post-irradiation annealing to take place. The fluence we refer is in $1-\mathrm{MeV}$ neutron equivalent value taking into account the NIEL factor of 1.4. The fluence uncertainty is determined by the ${ }^{27} \mathrm{Al}(\mathrm{pn})$ reaction cross section, which is no more than $10 \%$.

\section{INTERSTRIP CAPACITANCE AND RESISTANCE MEASUREMENTS}

Interstrip resistance measurements were performed in a probe station. As seen in Fig. 2, the DC pad of a test strip was connected to an Agilent 4156C Precision Semiconductor

Parameter Analyzer and grounded while a voltage $V_{2}$ was

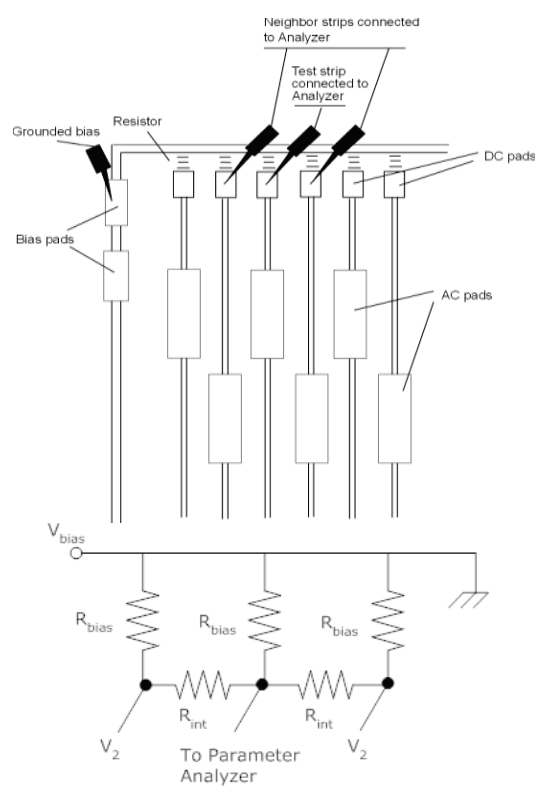

Fig. 2. Measurement set-up for interstrip resistance. Visual illustration of the measurement (top), showing the connections to the strips via the DC pads and to the bias ring. An equivalent circuit diagram (bottom) is also displayed.

applied to the DC pads of two of its closest neighbors, which were also connected to the analyzer. The detectors were biased using a Keithley 2410 HV Source Meter and each measurement were performed at several bias voltages ranging from $5 \mathrm{~V}$ to $300 \mathrm{~V}$. The voltage $\mathrm{V}_{2}$ applied to the neighbor strips was varied through the parameter analyzer from $-1 \mathrm{~V}$ to $1 \mathrm{~V}$ in $100 \mathrm{~mW}$ steps. Each measurement was performed at $22^{\circ}$ $\mathrm{C}$ with nitrogen gas flowing over the detector for moisture control.

The idea is that the voltage $\mathrm{V}_{2}$ on the neighbors will induce a current $I_{1}$ through the test strip. The interstrip resistance can then be determined by

$$
R_{\text {int }}=2 d V_{2} / d I_{1}
$$

where the factor of 2 comes from the fact that two neighboring strips are used. The resulting IV curve is shown in Fig. 3.

To measure the interstrip capacitance a slightly different setup was used. Instead of using the DC pads, the test strip and its neighbors were connected to an Agilent E4980A Precision LCR meter via the AC pads. In addition, the next two neighbors (two strips away from the test) were grounded to act as a shield from the outer laying strips. The frequency of the AC signal from the LCR meter was varied, and each measurement was taken at $10 \mathrm{kHz}, 100 \mathrm{kHz}, 1 \mathrm{MHz}$ and 2 $\mathrm{MHz}$. The bias voltage ranged from 0 to $-800 \mathrm{~V}$ with a step size of $50 \mathrm{~V}$. The measurements were again performed at $22^{\circ}$ $\mathrm{C}$ with nitrogen gas flowing over the detector.

\section{PunCh-Through Protection MeAsurements}

Protection against large voltages between strip implants and the metal traces can be achieved with the punch-through effect between strips and bias ring. This is trivially done for $\mathrm{p}$ spray, but requires sophisticated structures in the case of $\mathrm{p}$ - 


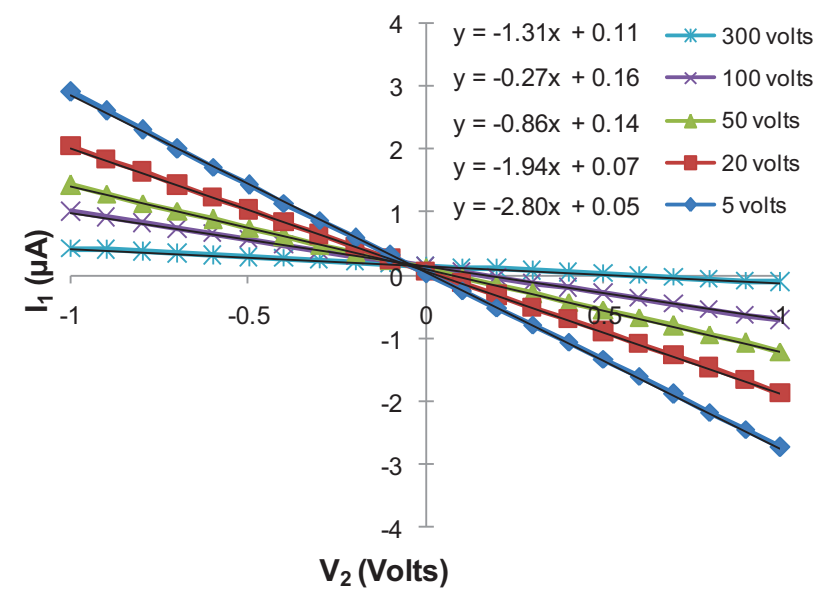

Fig. 3. The current through the test strip vs. the voltage applied to the neighbors shown at different bias votlages. The interstrip resistance is given by the inverse of the slope.

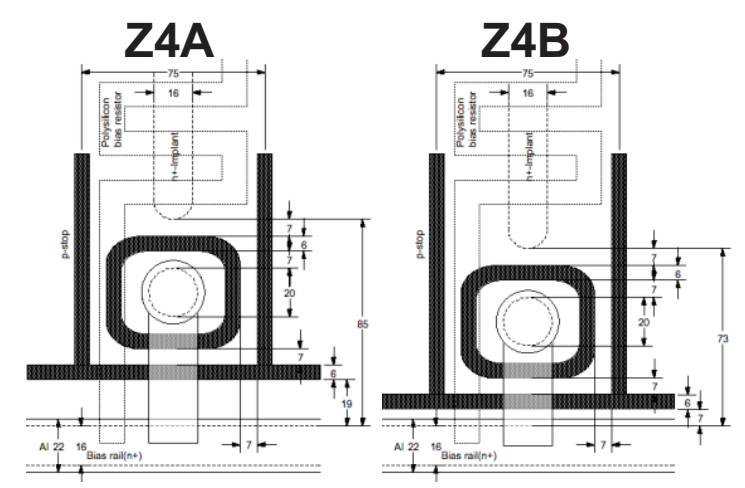

Z4C Z4D

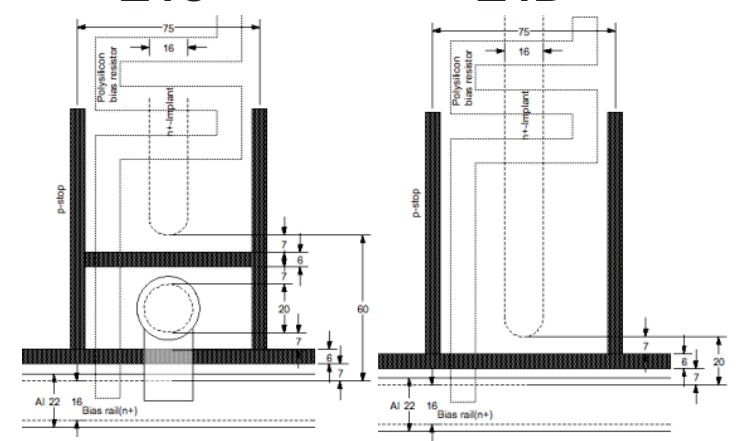

Fig. 4. The different PTP structures designs that have been tested for the paper.

stops. In this paper, the effects of several PTP structures on the PTP voltage have been studied. In particular, zone 4 detectors were designed with complicated PTP structures, as seen in Fig. 4.

Unirradiated detectors were measured at Lancaster University in a probe station kept at $21^{\circ} \mathrm{C}$. Measurements on irradiated detectors were carried out at the University of Tsukuba where detectors were placed two at a time on printed circuit boards which were then placed in a thermostat chamber set at $-20^{\circ} \mathrm{C}$ with nitrogen gas flowing in for moisture control.
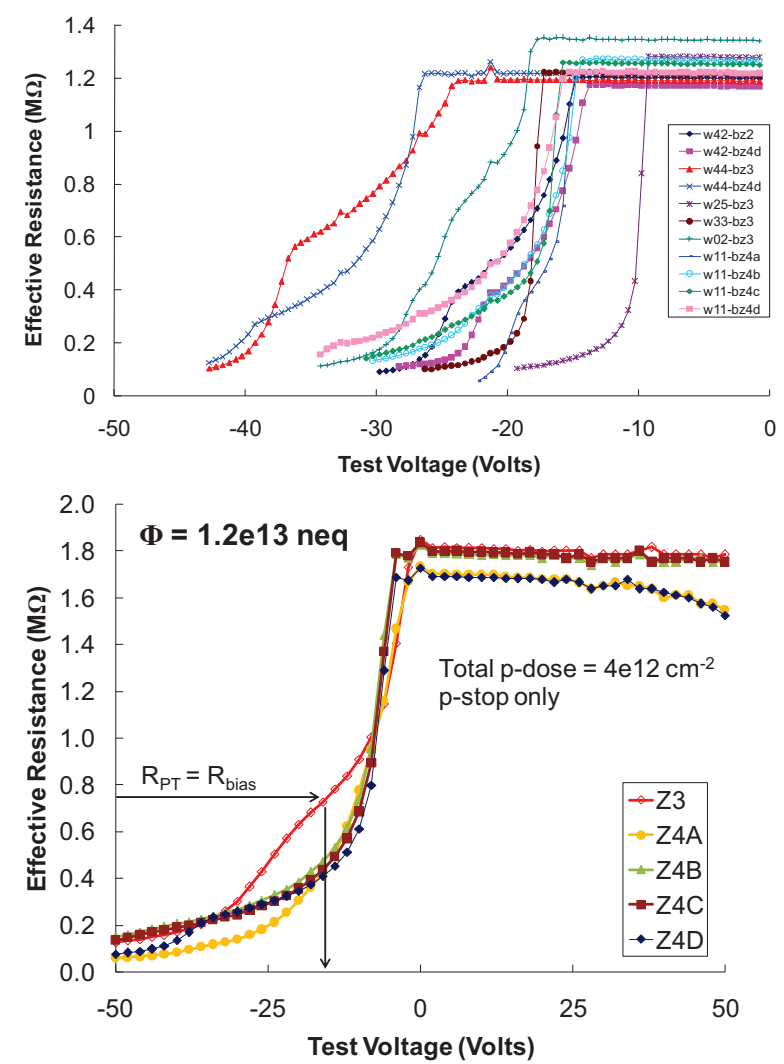

Fig. 5. The effective resistance vs. the test voltage applied for a) unirradiated detectors and for $\mathrm{b}$ ) detectors irradiated with protons to $1.2 \times 10^{13}$ neq. The horizontal arrow indicates where the punch-through resistance is equal to the value of bias resistor, and the vertical arrow indicates the corresponding punch-through voltage.

For PTP measurements, the effective resistance is measured between the DC pad of a strip and the bias ring. The detectors were reversed biased to $300 \mathrm{~V}$ with voltage being applied to the backplane and the bias ring grounded. A test voltage $\mathrm{V}_{\text {test }}$ is then applied to the DC pad, and the subsequent induced $\mathrm{I}_{\text {test }}$ current was measured between the pad and the bias ring. The neighboring strips were left floating. The effective resistance is then given by

$$
R_{e f f}=d V_{\text {test }} / d I_{\text {test }}
$$

The effective resistance can be thought of as the bias resistor in parallel to another resistance, which we call the punch-through resistance. The effective resistance can then be written as

$$
R_{e f f}=\left(1 / R_{\text {bias }}+1 / R_{P T}\right)^{-1}
$$

where $R_{\text {bias }}$ is value of the bias resistor and $R_{P T}$ is the punchthrough resistance.

The punch-through voltage $V_{\mathrm{PT}}$ is then taken to be the voltage where

$$
R_{P T}=R_{\text {bias }}
$$




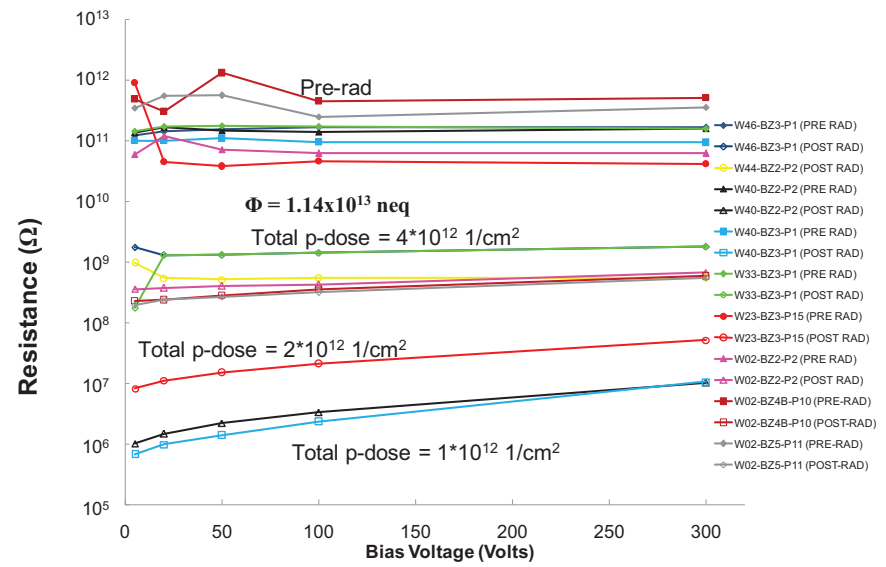

Fig. 6. Interstrip resistance for irradiated series 3 detectors. There is a clear dependence on the total p-dose applied after irradiation.

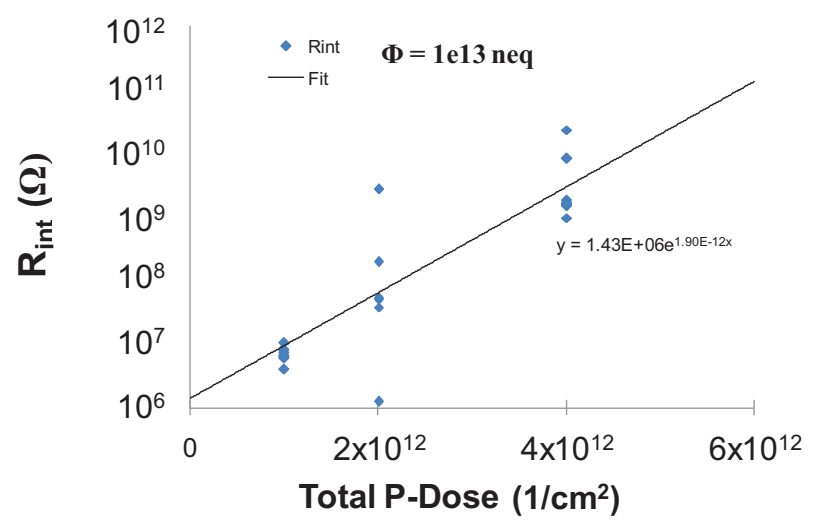

Fig. 7. Interstrip resistance vs. the total p-dose applied for detectors irradiated with protons to $1 \times 10^{13}$ neq. Higher $\mathrm{p}$-doses lead to higher interstrip resistance after irradiation.

or alternatively the point where the effective resistance is equal to half the value of the bias resistor The effective resistance as a function of the test voltage for an unirradiated detector is shown in Fig. 5a while irradiated detectors are shown in Fig. 5b.

\section{RESULTS}

To first order, the interstrip resistance does not seem to depend on the specific zone, but instead depends only on the total amount of $\mathrm{p}$-dose applied to the surface (through $\mathrm{p}$-stop or $\mathrm{p}$ spray), as seen in Fig. 6. There is an obvious correlation between the total amount of p-dose applied and the value of interstrip resistance after irradiation, with a higher total p-dose resulting in better post-rad strip isolation (higher interstrip resistance), which is illustrated in Fig. 7.

The interstrip capacitance shows little change after irradiation, and seems to be dependent on the specific zone, but not really on the amount of total p-dose applied. Still, the dependence is weak, and no predictions could be made about whether adding a p-stop mask would increase or decrease, or by which amount, the interstrip capacitance. Zone 5 is the only exception to this, consistently showing an increase in capacitance after irradiation.

It is helpful to plot the interstrip resistance vs. the interstrip capacitance. This helps determine which detectors have the most favorable behavior (highest resistance and lowest capacitance). Detectors performing the best should lie in the upper-left corner

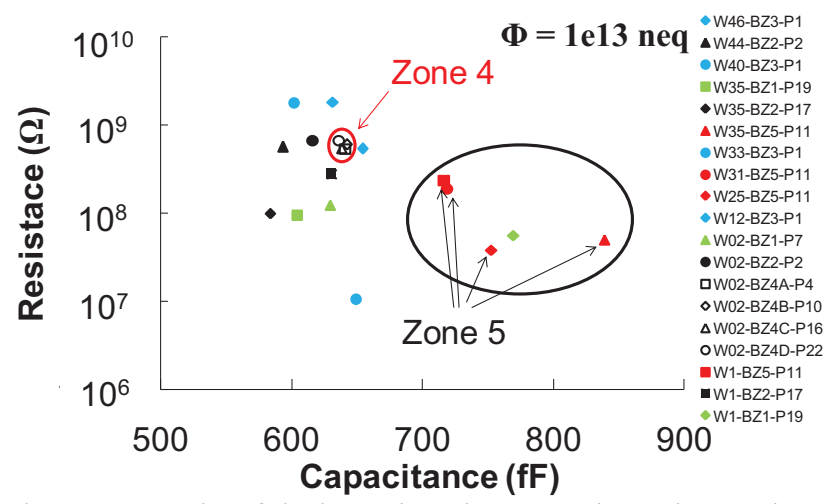

Fig. 8. Scatter plot of the interstrip resistance vs. interstrip capacitance for detectors irradiated to $1 \times 10^{13}$. Zone 5 (black circle) tends to perform the worst compared to the other zones.

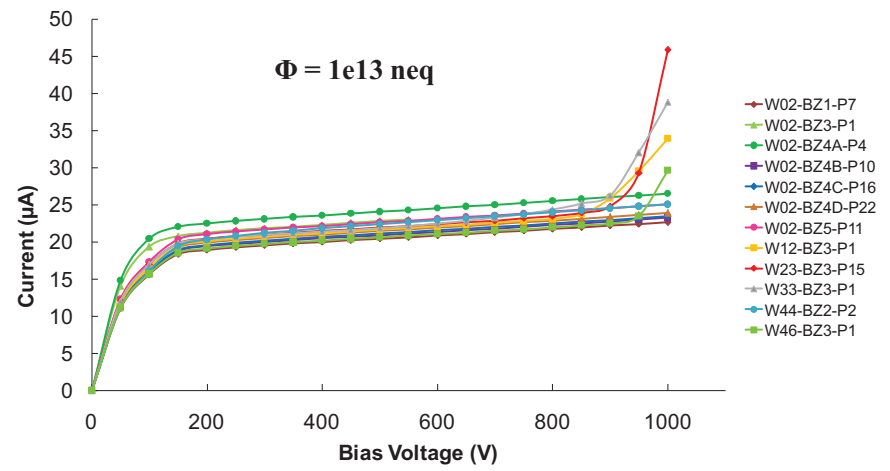

Fig. 9. Leakage current vs. bias voltage for various detector types irradiated to $1 \times 10^{13}$ neq taken at $22^{\circ} \mathrm{C}$. Zone 3 breaks down the earliest at $\sim 900 \mathrm{~V}$, which is still greater than the $600 \mathrm{~V}$ breakdown specified by the manufacturer.

of the graph, while the least preferable behavior is seen in the lower-right of the graph. Fig. 8 shows the interstrip resistance vs. capacitance for several detectors of different zones and total pdose. One can see that zone 5 tends to have the worst interstrip properties after irradiation.

The leakage current as a function of the bias voltage was measured in order to determine if good strip isolation came with the advantage of a lower breakdown voltage. The IV curves for various irradiated detectors taken at room temperature are shown in Fig. 9. It can be seen that all most detectors have a breakdown voltage in excess of $1000 \mathrm{~V}$, while three detectors (all zone 3) exhibit breakdown at about $900 \mathrm{~V}$. The high breakdown voltage should not significantly affect our measurements, as the interstrip resistance is taken at $300 \mathrm{~V}$, and the capacitance at $800 \mathrm{~V}$. It is also important to note that all detectors exhibit breakdown at a much higher voltage than the breakdown voltage of $600 \mathrm{~V}$ that was specified by the manufacturer.

The punch-through voltage for unirradiated detectors is between $10-30 \mathrm{~V}$, as seen in Fig. 10a. There seems to be a clear wafer correlation on the value of the punch-through voltage, i.e. the voltage depends on the total p-dose and type (p-spray, p-stop). After irradiation, a dependence of the punch-through voltage on the total p-dose and type is still evident, which is seen in Fig. $10 \mathrm{~b}$.

It is interesting to note that the punch-through voltage for zone 3 detectors and zone 4 detectors is similar before and after irradiation. This implies that the complicated punch-through protection structures are not needed, as zone 3 , which has a distance of $70 \mu \mathrm{m}$ from the $\mathrm{n}+$ implant to the bias rail, seems to provide adequate protection. Further, as seen in Fig. 11, zone 3 

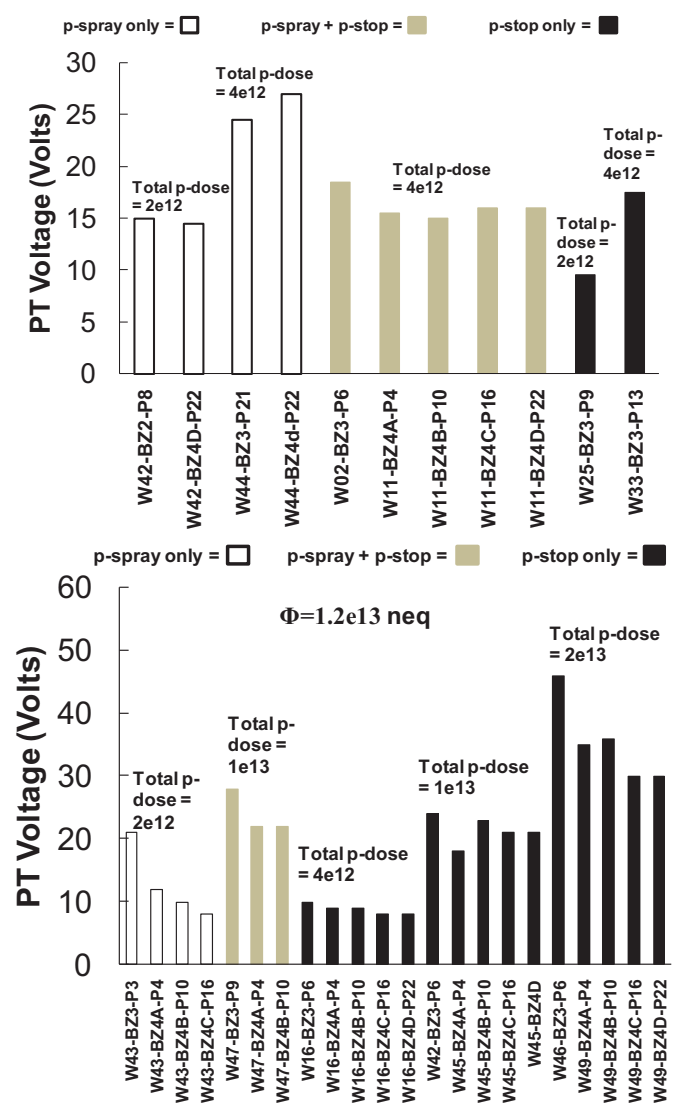

Fig. 10. Punch-through voltage for a) unirradiated detectors and b) detectors irradiated to $1.2 \times 10^{13}$ neq. Both pre and post-rad detectors show a dependence on the total p-dose applied.

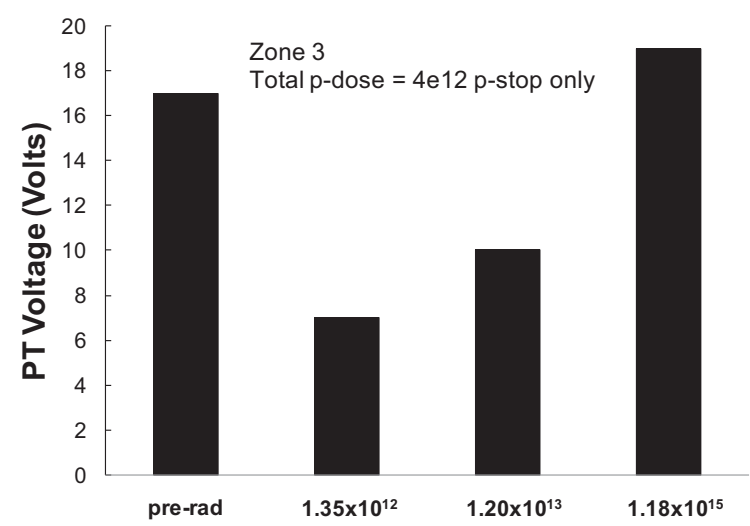

Fig. 11. Punch-through voltage for zone 3 measured at various fluences. Total $\mathrm{p}$-dose $=4 \times 10^{12} \mathrm{~cm}^{-2}$, fluence values are in neq.

detectors exhibit adequate protection even at high fluences, having a punch-through voltage in the range of $7-20 \mathrm{~V}$. This further suggests that complicated punch-through protection structures are not necessary

Measurements of the effective resistance taken at different temperatures indicate that there is clear temperature dependence, as seen in Fig. 12. The temperature dependence of the effective resistance would at first seem to complicate the extraction of the punch-through voltage, but it has been found that although the resistance is dependent on temperature, the value of the punchthrough voltage is independent of the temperature. The dependence on temperature seems to come mostly from the polysilicon bias resistor.

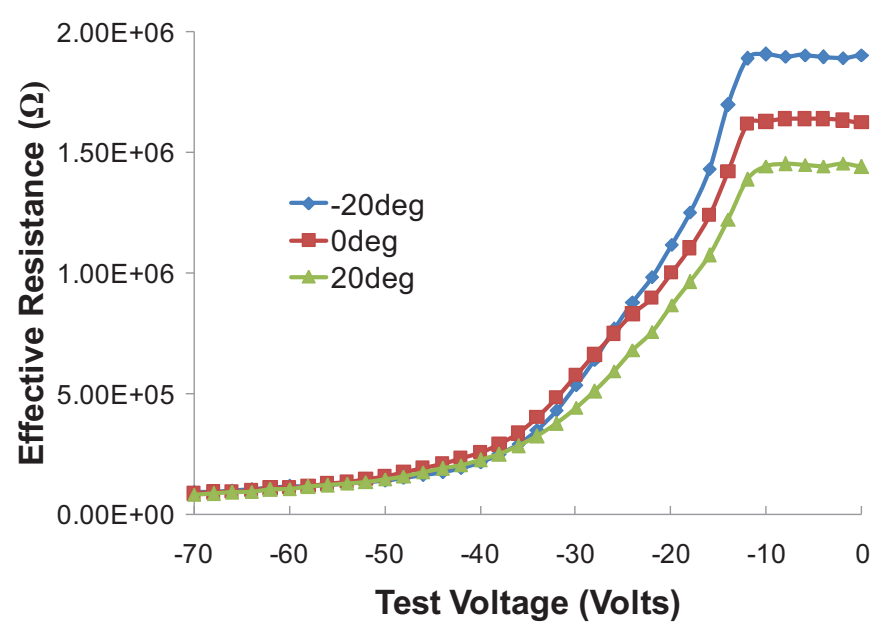

Fig. 12. The effective resistance measured at three different temperatures. There is a clear dependence on the temperature.

The effective resistance $R_{\text {eff }}$ can also be defined by taking the integral form of equation 2. The integral definition would have the added benefit of incorporating the total current that can be drained from the strip to the bias rail and providing the effective resistance for charges to escape through. As can be seen from Fig 13 , the disadvantage to this definition is that it is less sensitive to the onset of punch through, so it is less suitable for defining the punch-through voltage. This leads to a higher punch-through voltage, culminating in differences between 5-30Volts from the definition used in this paper.

\section{CONCLUSIONS AND SUMMARY}

The interstrip resistance decreases and interstrip capacitance increases after irradiation. To first order, the interstrip resistance does not depend on the specific zone of the detector, but instead depends on the total dose of the p-impurities applied. The higher the total dose of impurities, the interstrip capacitance shows little change after irradiation and is dependent on the specific zone. Specifically, zone 5, which has narrow metal, tends to have the highest interstrip capacitance after irradiation, without providing any benefits such as a higher breakdown voltage. The higher interstrip capacitance for zone 5 is not surprising given the shorter distance between readout strips compared to other zones.

Even if the interstrip resistance is low, one would only expect to see a loss in signal and increase in noise if the values were on the same order of magnitude as input impedance of the signal readout chip, which is typically about $1 \mathrm{k} \Omega$. The signal gets coupled to the metal strip and to the amplifier instead of being shorted to the neighbor via the DC conductance. So as long as the interstrip resistance is large compared to the input impedance of the chip, the signal will end up in the amplifier. Further, there has not yet been any observed signal degradation or noise increase due to a decrease in the interstrip resistance.

The leakage current for zone 3 detectors tends to start increasing at slightly lower voltages than other detectors, but the voltage is above $900 \mathrm{~V}$ and is much greater than the $600 \mathrm{~V}$ breakdown voltage specified by the manufacture.

Measurements of the punch-through voltage reveal that there is a clear wafer correlation. In particular, when looking at a 


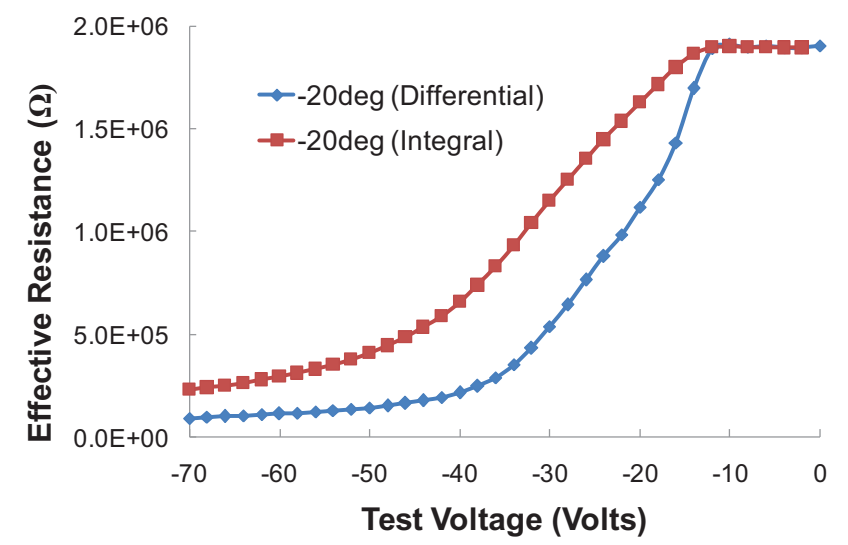

Fig. 13. Different definitions for the effective resistance. The integral definition (red curve) gives a higher punch-through voltage than the differential definition (blue curve).

specific configuration ( $\mathrm{p}$-spray only, $\mathrm{p}$-stop only, $\mathrm{p}$-spray+stop) wafers with the highest $\mathrm{p}$-dose have the highest punch-through voltage, which holds true even after irradiation.

After irradiation, zone 3 detectors have similar punch-through voltages as zone 4 detectors, which have a much more complicated punch-through protection structure. The acceptable punch-through voltage of zone 3 detectors with p-stops of $4 \times 10^{12}$ $\mathrm{cm}^{-2}$ extends to proton fluences beyond $10^{15} \mathrm{p} / \mathrm{cm}^{2}$. Thus, punchthrough protection is achievable with zone 3 which has no dedicated structure.

There is a clear dependence of the effective resistance on temperature. Measurements at different temperatures do not lead to different values for the punch-through voltage. In fact the temperature dependence seems to come entirely from the bias resistor.

It is important to mention that equation (2) is not the only way to define $R_{\text {eff. }}$ An alternate definition would be to take the integral form of equation (2). This would have the advantage of incorporating the total current that can be drained from the strip to the bias rail and providing the effective resistance for charges to escape through. But the integral definition is less sensitive to the onset of punch-though, and thus less suitable for finding the onset of the punch-though voltage.

\section{REFERENCES}

[1] M. Bruzzi, H. F.-W. Sadrozinski, A. Seiden "Comparing radiation tolerant materials and devices for ultra rad-hard tracking detectors." Nucl. Inst. Meth. A 579 (2007) 754-761.

[2] F. Giannotti et al., "Physics potential and experimental challenges of the LHC luminosity upgrade", hep-ph/0204087, April 2002.

[3] ATLAS Radiation Taskforce Report.

http://atlas.web.cern.ch/GROUPS/PHYSICS/RadiationTF_document.html

[4] M. Bruzzi, IEEE Trans. Nucl. Sci., 48, 4 (2001).

[5] http://rd50.web.cern.ch/rd50/

[6] J. Ellison et al, IEEE Trans. Nucl. Sci. 36-1 (1989) 267-271

[7] K. Hara et al., IEEE Trans. Nucl. Sci., 56-2 (2009) 468.

[8] H. F. -W. Sadrozinski et al., Nucl. Instr. and Meth. A 579 (2007) 769774.

[9] Y. Unno et al., Nucl. Instr. and Meth. A 579 (2007) 614. 